\title{
Transcript analysis of a goat mesenteric lymph node by deep next-generation sequencing
}

\author{
G.X. E, Y.J. Zhao, R.S. Na and Y.F. Huang \\ Chongqing Key Laboratory of Forage \& Herbivore, \\ Chongqing Engineering Research Centre for Herbivores Resource Protection \\ and Utilization, College of Animal Science and Technology, \\ Southwest University, Chongqing, China \\ Corresponding author: Y.F. Huang \\ E-mail: H67738337@swu.edu.cn
}

Genet. Mol. Res. 15 (2): gmr.15028262

Received December 14, 2015

Accepted February 26, 2016

Published April 29, 2016

DOI http://dx.doi.org/10.4238/gmr.15028262

\begin{abstract}
Deep RNA sequencing (RNA-seq) provides a practical and inexpensive alternative for exploring genomic data in non-model organisms. The functional annotation of non-model mammalian genomes, such as that of goats, is still poor compared to that of humans and mice. In the current study, we performed a whole transcriptome analysis of an intestinal mucous membrane lymph node to comprehensively characterize the transcript catalogue of this tissue in a goat. Using an Illumina HiSeq 4000 sequencing platform, 9.692 GB of raw reads were acquired. A total of 57,526 lymph transcripts were obtained, and the majority of these were mapped to known transcriptional units $(42.67 \%)$. A comparison of the mRNA expression of the mesenteric lymph nodes during the juvenile and post-adolescent stages revealed 8949 transcripts that were differentially expressed, including 6174 known genes. In addition, we functionally classified these transcripts using Gene Ontology (GO) and the Kyoto Encyclopedia of Genes and Genomes (KEGG) terms. A total of 6174 known genes were assigned to $64 \mathrm{GO}$ terms, and 3782 genes were assigned to $303 \mathrm{KEGG}$ pathways, including some related to immunity. Our results reveal the complex
\end{abstract}


transcriptome profile of the lymph node and suggest that the immune system is immature in the mesenteric lymph nodes of juvenile goats.

Key words: Transcriptome; RNA-seq; Lymph; Goat

\section{INTRODUCTION}

The mucosal immune system (MIS) is the part of the immune system that protects the mucous membranes of organisms from invasion by potentially pathogenic microbes. The MIS has three main functions: protection of the mucous membrane against infection; preventive uptake of antigens, microorganisms, and other foreign materials; and moderation of the organism's immune response to such materials (Holmgren and Czerkinsky, 2005).

Mucosal-associated lymphoid tissue (MALT) is composed of eight parts, including gut-associated lymphoid tissue (GALT), bronchus-associated lymphoid tissue (BALT), nose-associated lymphoid tissue (NALT), conjunctival-associated lymphoid tissue (CALT), organized mucosa-associated lymphatic tissue (O-MALT), diffuse mucosa-associated lymphatic tissue (D-MALT), larynx-associated lymphoid tissue (LALT), and skin-associated lymphoid tissue (SALT). At birth, the neonate's mucosal immune system is relatively undeveloped, but the colonization of intestinal flora accelerates its development. Because of its frontline status within the immune system, the mucosal immune system is being investigated for use in vaccines for various diseases (Bourinbaiar et al., 2003; Simerska et al., 2009; Pavot et al., 2014). There are far more studies on lymph immunology in domestic animals than in peripheral model organs and humans, particularly for the intestinal mucosa, which is the largest immunologic organ (Esfandiari et al., 2014).

Deep RNA sequencing (RNA-seq) is an effective method to identify gene expression profiles and mRNA-based biomarkers. Due to advancements in high-throughput technology, research has demonstrated that mRNA is actively transcribed in the eukaryotic genome and plays an important role in all aspects of normal biological functions (e.g., Bao et al., 2014; Fang et al., 2015); particularly immunological functions (Malecki and Szybalski, 2012; Miller et al., 2012). High-throughput technology has been widely applied to research in domestic animals, providing abundant resources for data mining (e.g., Cao et al., 2014; Zhao et al., 2015).

Goats (Capra hircus) are domesticated animals that are commonly found throughout the tropical and subtropical regions around the world. The goat is a predominantly domestic animal that provides not only meat but also cashmere, milk, wool, and fur. Investigation of its developmental mechanisms and a deeper understanding of the physiological mechanism of its mesenteric lymph nodes is important for increasing the survival rate of goats in agriculture. In this study, we profiled the gene expression of goat mesenteric lymph nodes using RNA re-sequencing technology and compared the protein-coding gene expression profiles of the mesenteric lymph nodes of adult and juvenile individuals. We also performed a functional annotation of the differentially expressed protein-coding genes.

\section{MATERIAL AND METHODS}

The experimental conditions of this study were approved by the Committee on the Ethics of Animal Experiments of Southwest University [No. (2007) 3] and the Animal Protection Law of China. 
A sample from one female Wutong black goat at 48 days of age (Sample_1) was collected on March 1, 2015. The sample was transported from the collection site in Chongqing, China $\left(108^{\circ} 03^{\prime} 25.85^{\prime \prime} \mathrm{E}, 30^{\circ} 53^{\prime} 26.64^{\prime \prime} \mathrm{N}, 1271 \mathrm{~m}\right)$ to a domestic animal conservation field at Southwest University (Chongqing, China; 106²5'29.19"E, 2949'36.72"N, $763 \mathrm{~m}$ ). The animal was raised until euthanization on March 15, 2015 (ref. Qiao et al., 2013). The collected tissue (intestinal mucous membrane lymph node) was stored in Sample Protector Solution (TaKaRa, Japan) immediately after removal.

Total RNA was extracted from the tissue using TRIzol ${ }^{\circledR}$ Reagent according the manufacturer protocol (Invitrogen, USA), and genomic DNA was removed using DNase I (TaKara, Japan). RNA quality was determined using a 2100 Bioanalyzer (Agilent) and quantified using the ND-2000 (NanoDrop Technologies). A high-quality RNA sample $\left(\mathrm{OD}_{260 / 280}=1.8-2.2, \mathrm{OD}_{260 / 230} \geq 2.0, \mathrm{RIN} \geq 6.5,28 \mathrm{~S}: 8 \mathrm{~S} \geq 1.0>10 \mu \mathrm{g}\right)$ was used to construct the sequencing library.

RNA-seq transcriptome libraries were prepared following the instructions for the TruSeq RNA Sample Preparation Kit from Illumina (San Diego, CA), using $5 \mu \mathrm{g}$ of total RNA. Briefly, mRNA was isolated by polyA selection using oligo (dT) beads and fragmented using fragmentation buffer. Next, cDNA synthesis, end repair, A-base addition, and ligation of the Illumina-indexed adaptors were performed according to the manufacturer protocol. After quantification by TBS380, paired-end libraries were sequenced on the Illumina HiSeq 4000 platform ( 2 x 151 bp read length). The control data from a sample of lymph tissue from a post-adolescent goat (Sample_2) was obtained from a published dataset (GSM920221) (Dong et al., 2013).

The raw paired-end reads were trimmed and quality controlled using SeqPrep (https:// github.com/jstjohn/SeqPrep) and Sickle (https://github.com/najoshi/sickle) with default parameters. The clean reads were separately aligned to the $C$. hircus reference genome (CHIR_1.0) (Dong et al., 2013) in orientation mode using Bowtie2 software (http://bowtiebio.sourceforge.net/index.shtml) (Langmead and Salzberg, 2013).

To identify the DEGs (differential expression genes) in the two different samples, the expression level for each transcript was calculated using the fragments per kilobase of exon per million mapped reads (FRKM) method. Cuffdiff (http://cufflinks.cbcb.umd.edu/) (Trapnell et al., 2013) was used for the differential expression analysis. The DEGs of the two samples were selected using the following criteria: 1) a logarithmic fold change greater than 2; and 2) a P value FDR (false discovery rate) of less than 0.05 . To understand the functions of the differentially expressed genes, GO functional enrichment and KEGG pathway analysis were carried out using Goatools (https://github.com/tanghaibao/Goatools) and KOBAS (http://kobas.cbi.pku.edu.cn/ home.do) (Xie et al., 2011). DEGs were considered to be significantly enriched in GO terms and metabolic pathways when their Bonferroni-corrected P value was less than 0.05.

\section{RESULTS AND DISCUSSION}

\section{Illumina sequencing and gene annotation}

Previous studies have examined differences in gene expression associated with different phenotypes in goats using microarray analysis (Zhu et al., 2014; Tang et al., 2015; Wang et al., 2015); however, no research has focused on lymph immunology and its development in domesticated animals. To further investigate genes that may play important roles in the development and function of the goat lymphatic system, particularly the mesenteric lymph 
node, the mRNA profile was obtained for one juvenile mesenteric lymph node.

Illumina sequencing of $C$. hircus yielded a total of 68,381,774 raw reads with 9.692 GB. The transcriptome sequencing data from the mesenteric lymph node of the goat was deposited in the NCBI Sequence Read Archive database (accession number: SRS1163816). A reference dataset was constructed for gene annotation, which included protein data from the goat genome (CHIR_1.0). A total of 62,088,060 clear reads with 8.270 GB were generated through Illumina paired-end sequencing. Transcripts were blasted against this dataset. In total, 24,547 of 57,526 transcripts were annotated to 8949 known genes, which comprised $42.67 \%$ of the total number of transcripts.

\section{DEGs between mesenteric lymph tissues}

In an analysis of the digital gene expression (DGE) of the lymph tissue in the juvenile and post-adolescent goats, we found 8949 differential transcripts, including 6174 known genes that were significantly differentially expressed. Of these, 3277 genes from the mesenteric lymph node were upregulated compared to the other dataset (Sample_2), and 2897 were downregulated (Figure 1). We acknowledge that the study design was not optimal due to a lack of replication, since single pooled samples were used for the transcriptome sequencing analysis, and the data were compared to the data from one post-adolescent lymph tissue sample (GSM920221) reported in a previous study (Dong et al., 2013). Despite these limitations, our results enhanced the understanding of juvenile goat mesenteric lymph node composition and potential differences in gene expression as compared to post-adolescent lymph tissue, laying the foundation for future studies.
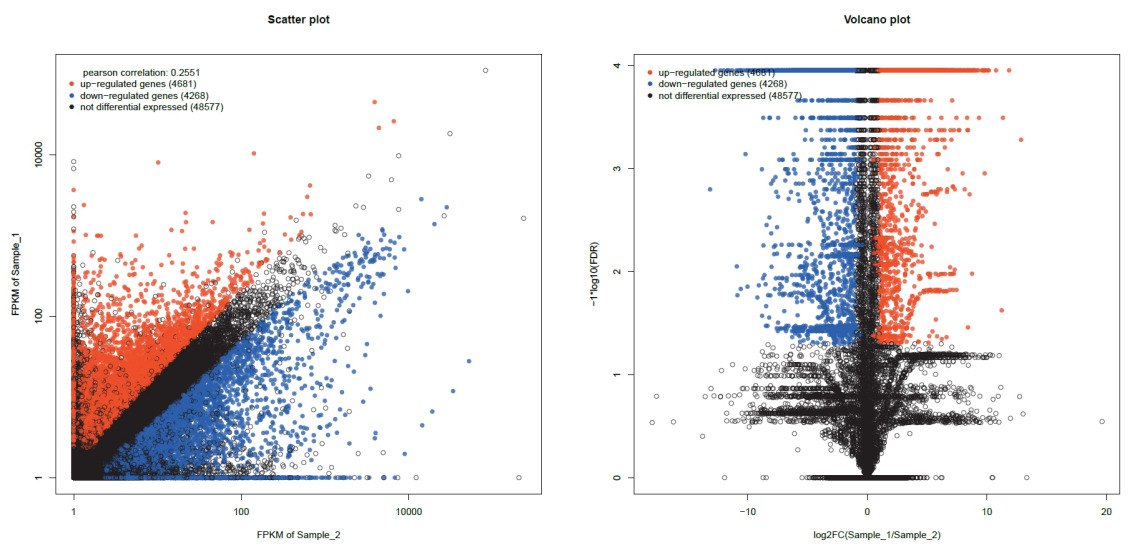

Figure 1. Identification of differentially expressed transcripts of lymph tissue in juvenile and post-adolescent goats.

\section{GO classification of unique transcripts in the mesenteric lymph node}

A GO analysis was performed to functionally classify the transcriptome sequences that had hits in the NCBI NR database. These were summarized under three main GO categories: cellular component, molecular function, and biological process (Figure 2). The 6174 DEGs were associated with 64 GO terms. Most of the DEGs could be classified into 
three GO categories (biological process, cellular component, and molecular function), which could then be used to implicate the biological processes involved in lymph node development.

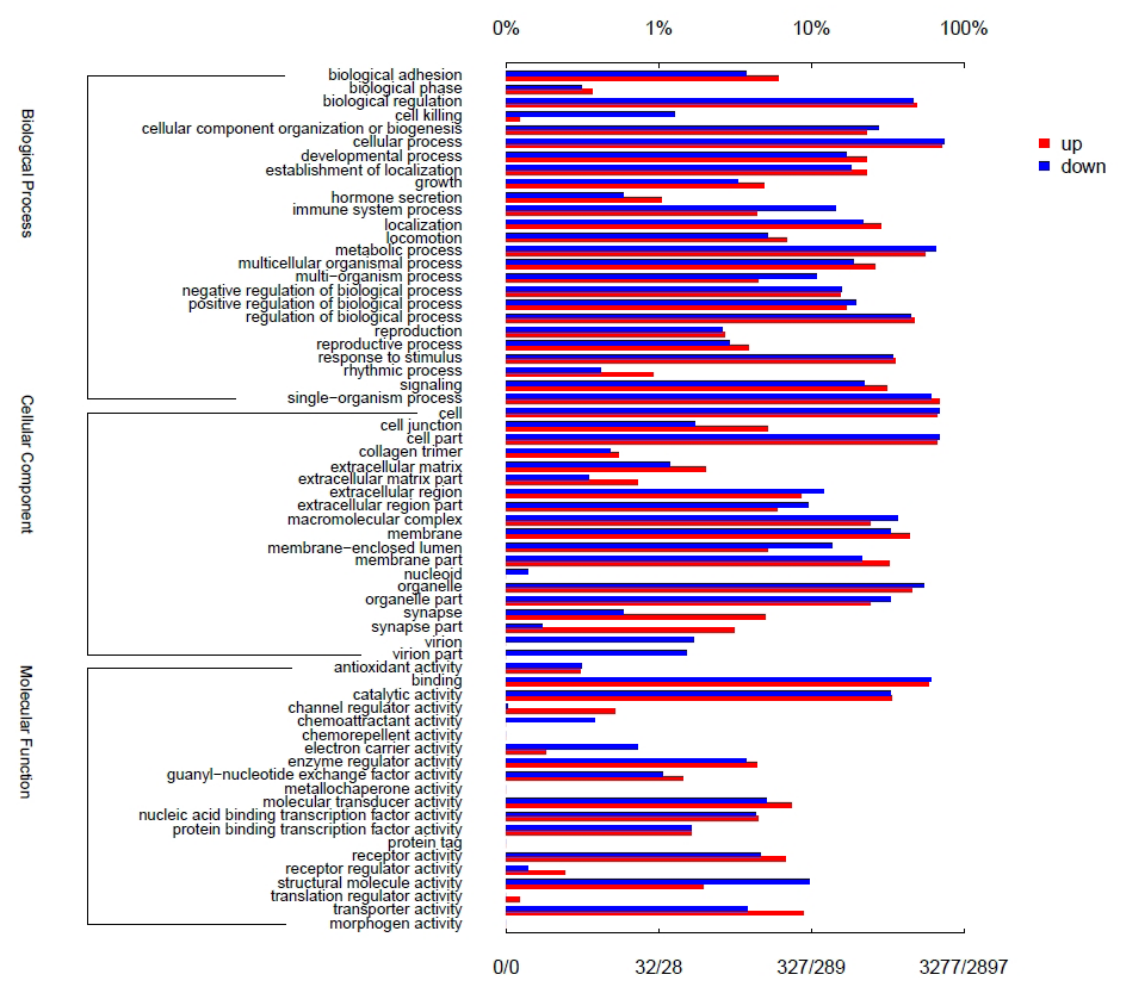

Figure 2. Distribution comparison of Gene Ontology (GO) categories of differentially expressed transcripts.

\section{KEGG analysis of unique transcripts in the mesenteric lymph node}

The KEGG database (Kanehisa and Goto, 2000) was used to identify potentially important biological pathways in our dataset. A total of 3782 genes were assigned to 303 KEGG pathways. Furthermore, most of the KEGG pathways involved immune function (e.g., the B-cell receptor signaling pathway, the Toll-like receptor signaling pathway, the intestinal immune network for IgA production, the NF-kappaB signaling pathway, the T cell receptor signaling pathway, and antigen processing and presentation), nervous system diseases (e.g., Huntington's disease, Alzheimer's disease, Parkinson's disease, and prion diseases) and cancer (e.g., pancreatic cancer, small cell lung cancer, pathways in cancer, and transcriptional misregulation in cancer). These annotations provide a valuable resource for investigating specific processes, functions, and pathways in lymph functional research.

Notably, two signaling pathways, antigen processing and presentation and the $\mathrm{T}$ cell receptor signaling pathway, were identified in our study. The T cell is a specific type of lymphocyte that plays an indispensable role in cell-mediated immunity (Figure $3 \mathrm{~A}$ ). This cell can be distinguished from other B cells and NK cells by the presence of the T cell receptor on the cell surface (Alberts et al., 2002; McClory et al., 2012). Different types of T cells 
participate in the adaptive and innate immune systems; e.g., alpha beta $\mathrm{T}$ cells in the former and gamma delta T cells in the latter (Vantourout and Hayday, 2013). However, many of the mediated factors in the $T$ cell receptor signaling pathway that were identified in the current study revealed an inactive stage of immune system function in the mesenteric lymph node of the young goat. The major histocompatibility complex (MHC) is a set of cell surface molecules that comprise a major part of the immune system by binding to the cell surface of pathogens for recognition by T-cells (Birnbaum et al., 2014). The gene expression pattern of the antigen processing and presentation signaling pathways (Figure 3B) suggested that genes of the MHC I and MHC II pathways were down regulated and expressed at low levels in the lymph tissue of the juvenile goat compared to that of the post-adolescent goat.
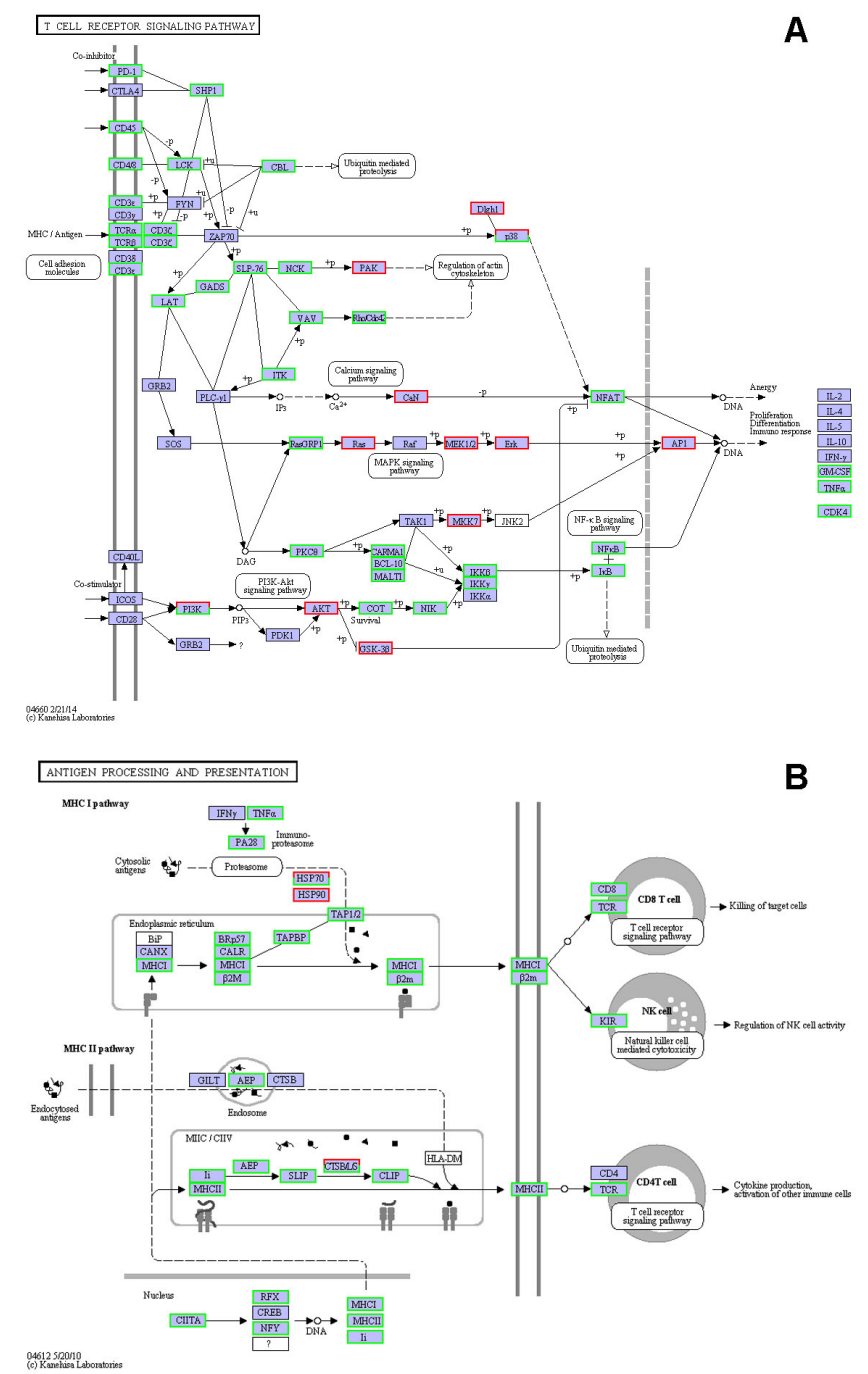

Figure 3. Differential expression of lymph tissue genes in the immunity-related pathway of juvenile and postadolescent goats. 


\section{CONCLUSION}

In summary, 57,526 transcripts were detected in the mesenteric lymph node of a goat using transcriptome sequencing. In total, 8945 transcripts were differentially expressed in juvenile and post-adolescent lymph tissue, of which 6174 known transcripts were assigned to GO categories and KEGG pathways. As a result, 6174 known DGE were mapped to 303 KEGG pathways and clustered into $3 \mathrm{GO}$ categories: biological process, cellular component, and molecular function. The results comparing the mRNA expression of the mesenteric lymph node of juvenile and post-adolescent goats suggested that during the development of the lymph node, the immune system functions were immature. These transcriptome data provide new resources for functional genome studies in future biological research involving $C$. hircus. Moreover, our findings are essential for increasing the understanding of the biological functions and developmental mechanisms of the mesenteric lymph node.

\section{Conflicts of interest}

The authors declare no conflict of interest.

\section{ACKNOWLEDGMENTS}

Research supported by the Fundamental Research Funds for the Central Universities (\#SWU114052), the National Natural Science Foundation of China (\#31172195), and the 2013 Innovation Team-Building Program in Chongqing Universities (\#KJTD201334).

\section{REFERENCES}

Alberts B, Johnson A, Lewis J, Raff M, et al. (2002). Molecular Biology of the Cell. Garland Science, New York.

Bao ZS, Chen HM, Yang MY, Zhang CB, et al. (2014). RNA-seq of 272 gliomas revealed a novel, recurrent PTPRZ1-MET fusion transcript in secondary glioblastomas. Genome Res. 24: 1765-1773 http://dx.doi.org/10.1101/gr.165126.113.

Birnbaum ME, Mendoza JL, Sethi DK, Dong S, et al. (2014). Deconstructing the peptide-MHC specificity of T cell recognition. Cell 157: 1073-1087 http://dx.doi.org/10.1016/j.cell.2014.03.047.

Bourinbaiar AS, Metadilogkul O and Jirathitikal V (2003). Mucosal AIDS vaccines. Viral Immunol. 16: 427-445 http:// dx.doi.org/10.1089/088282403771926274.

Cao S, Han J, Wu J, Li Q, et al. (2014). Specific gene-regulation networks during the pre-implantation development of the pig embryo as revealed by deep sequencing. BMC Genomics 15: 4 http://dx.doi.org/10.1186/1471-2164-15-4.

Dong Y, Xie M, Jiang Y, Xiao N, et al. (2013). Sequencing and automated whole-genome optical mapping of the genome of a domestic goat (Capra hircus). Nat. Biotechnol. 31: 135-141 http://dx.doi.org/10.1038/nbt.2478.

Esfandiari NH, Hughes DT, Yin H, Banerjee M, et al. (2014). The effect of extent of surgery and number of lymph node metastases on overall survival in patients with medullary thyroid cancer. J. Clin. Endocrinol. Metab. 99: 448-454 http://dx.doi.org/10.1210/jc.2013-2942.

Fang SM, Hu BL, Zhou QZ, Yu QY, et al. (2015). Comparative analysis of the silk gland transcriptomes between the domestic and wild silkworms. BMC Genomics 16: 60 http://dx.doi.org/10.1186/s12864-015-1287-9.

Holmgren J and Czerkinsky C (2005). Mucosal immunity and vaccines. Nat. Med. 11 (Suppl): S45-S53 http://dx.doi. org $/ 10.1038 / \mathrm{nm} 1213$.

Kanehisa M and Goto S (2000). KEGG: kyoto encyclopedia of genes and genomes. Nucleic Acids Res. 28: 27-30 http:// dx.doi.org/10.1093/nar/28.1.27.

Langmead B and Salzberg SL (2013). Fast gapped-read alignment with Bowtie 2. Nat. Methods 9: 357-359. http://dx.doi. org/10.1038/nmeth.1923

Malecki M and Szybalski W (2012). Isolation of single, intact chromosomes from single, selected ovarian cancer cells for in situ hybridization and sequencing. Gene 493: 132-139 http://dx.doi.org/10.1016/j.gene.2011.11.044. 
McClory S, Hughes T, Freud AG, Briercheck EL, et al. (2012). Evidence for a stepwise program of extrathymic T cell development within the human tonsil. J. Clin. Invest. 122: 1403-1415 http://dx.doi.org/10.1172/JCI46125.

Miller LC, Fleming D, Arbogast A, Bayles DO, et al. (2012). Analysis of the swine tracheobronchial lymph node transcriptomic response to infection with a Chinese highly pathogenic strain of porcine reproductive and respiratory syndrome virus. BMC Vet. Res. 8: 208 http://dx.doi.org/10.1186/1746-6148-8-208.

Pavot V, Rochereau N, Lawrence P, Girard MP, et al. (2014). Recent progress in HIV vaccines inducing mucosal immune responses. AIDS 28: 1701-1718. http://dx.doi.org/10.1097/QAD.0000000000000308

Qiao L, Yang W, Fu J and Song Z (2013). Transcriptome profile of the green odorous frog (Odorrana margaretae). PLoS One 8: e75211 http://dx.doi.org/10.1371/journal.pone.0075211.

Simerska P, Moyle PM, Olive C and Toth I (2009). Oral vaccine delivery--new strategies and technologies. Curr. Drug Deliv. 6: 347-358. http://dx.doi.org/10.2174/156720109789000537

Tang Q, Huang W, Guan J, Jin L, et al. (2015). Transcriptomic analysis provides insight into high-altitude acclimation in domestic goats. Gene 567: 208-216 http://dx.doi.org/10.1016/j.gene.2015.05.007.

Trapnell C, Hendrickson DG, Sauvageau M, Goff L, et al. (2013). Differential analysis of gene regulation at transcript resolution with RNA-seq. Nat. Biotechnol. 31: 46-53. http://dx.doi.org/10.1038/nbt.2450

Vantourout P and Hayday A (2013). Six-of-the-best: unique contributions of gd T cells to immunology. Nat. Rev. Immunol. 13: 88-100 http://dx.doi.org/10.1038/nri3384.

Wang YH, Zhang CL, Plath M, Fang XT, et al. (2015). Global transcriptional profiling of longissimus thoracis muscle tissue in fetal and juvenile domestic goat using RNA sequencing. Anim. Genet. 46: 655-665 http://dx.doi.org/10.1111/ age. 12338 .

Xie C, Mao X, Huang J, Ding Y, et al. (2011). KOBAS 2.0: a web server for annotation and identification of enriched pathways and diseases. Nucleic Acids Res. 39: W316-W322. http://dx.doi.org/10.1093/nar/gkr483

Zhao ZQ, Wang LJ, Sun XW, Zhang JJ, et al. (2015). Transcriptome analysis of the Capra hircus ovary. PLoS One 10: e0121586 http://dx.doi.org/10.1371/journal.pone.0121586.

Zhu B, Xu T, Zhang Z, Ta N, et al. (2014). Transcriptome sequencing reveals differences between anagen and telogen secondary hair follicle-derived dermal papilla cells of the Cashmere goat (Capra hircus). Physiol. Genomics 46: 104111 http://dx.doi.org/10.1152/physiolgenomics.00132.2013. 